\title{
Multi-Objective Optimisation of Constellation Deployment Using Low-Thrust Propulsion
}

\author{
Marilena Di Carlo*, Lorenzo Ricciardi* and Massimiliano Vasile ${ }^{\dagger}$ \\ Department of Mechanical \& Aerospace Engineering, University of Strathclyde, Glasgow, UK
}

\begin{abstract}
This work presents an analysis of the deployment of future constellations using a combination of low-thrust propulsion and natural dynamics. Different strategies to realise the transfer from the launcher injection orbit to the constellation operational orbit are investigated. The deployment of the constellation is formulated as a multi-objective optimisation problem that aims at minimising the maximum transfer $\Delta V$, the launch cost and maximise at the same time the pay-off given by the service provided by the constellation. The paper will consider the case of a typical constellation with 27 satellites in Medium Earth Orbit and the use of only two launchers, one of which can carry a single satellite. It will be demonstrated that some strategies and deployment sequences are dominant and provide the best trade-off between peak transfer $\Delta V$ and monetary pay-off.
\end{abstract}

\section{Nomenclature}

a Semimajor axis

$i \quad$ Inclination

$\Omega \quad$ Right ascension of the ascending node

$\mu \quad$ Earth's gravitational parameter

$R_{\oplus} \quad$ Earth's radius

$J_{2} \quad$ Second zonal harmonic of the Earth's gravitational potential

$f \quad$ Acceleration of the low-thrust engine

$\beta \quad$ Elevation angle of the low-thrust vector

$\alpha \quad$ Semi-amplitude of the thrust arcs

ToF Time of flight

$\Delta V$ Variation of velocity required for the transfer

\section{Introduction}

Satellite constellations are used for a wide range of applications and are deployed in different orbital regime around the Earth, according to their purpose. Navigation systems such as the Global Positioning System (GPS), the Russian Glonass and the Chinese BiDou are based on satellite constellations in Medium Earth Orbit (MEO). The European Space Agency is currently launching its own navigation system, Galileo, in MEO. Telecommunication services are provided by constellations in Low Earth Orbit (LEO) such as Globalstar and Iridium. Constellations are present in Sun-Synchronous Orbits for Earth Observation purposes (A-Train) and in Geosynchronous High-Elliptic Orbit (HEO) for communications service to high-latitude regions (Sirius). More constellation services are going to be launched in the near future: as an example, OneWeb LLC plans to put 720 small satellites in LEO starting in 2017 to provide broadband services.

This trend demands for an efficient satellite constellation launch and deployment strategy.

*PhD candidate, Department of Mechanical \& Aerospace Engineering, University of Strathclyde, 75 Montrose Street, Glasgow, G1 1XJ, UK.

${ }^{\dagger}$ Professor, Department of Mechanical \& Aerospace Engineering, University of Strathclyde, 75 Montrose Street, Glasgow, G1 1XJ, UK. 
As the number of satellites in orbit around the Earth increases it is also paramount to devise an appropriate de-orbiting strategy for the spacecraft at the end of the constellation lifetime. The aim is to avoid what happened on February 10, 2009, when an inactive Russian communications satellite, Cosmos 2251, collided with one of the satellite of the Iridium constellation, producing almost 2,000 pieces of debris. It is therefore desirable to equip future constellations with a propulsion system with sufficient propellant to de-orbit at the end of life or move to a safe graveyard orbit.

In this work, we study the deployment of a constellation using low-thrust propulsion. The interest is in finding an optimal deployment sequence (which satellite is allocated to which slot), optimal launch sequence (which satellites are launched with which launcher) and optimal transfer strategy (which lowthrust trajectory is required to achieve the required slot) that can provide maximum pay-off, minimum launch cost and minimise the mass of propellant. The first objective defines the monetary gain provided by the service delivered by the constellation. The earlier the satellites in the constellation start providing their service the higher is the pay-off is. The last objective, the propellant mass, is dictating the sizing of the propulsion system and the mass allocated to the deployment sequence.

The deployment sequence is a complex combination problem that is here addressed with a simple deterministic greedy incremental algorithm that provides fast, though suboptimal, solutions. A separate combiantorial problem, equivalent to a bin packing problem, is solved to identify all the possible launch sequences assuming only two launchers are available. The solution of this second combinatorial problem provides the cost of the launch sequence. Finally the transfer strategy is optimised with a memetic evolutionary algorithm, called MACS2 (Multi-Agent Collaborative Search) that maximises the pay-off and minimises the maximum $\Delta V$ of the transfer. The $\Delta V$ costs of the transfers are calculated with a set of simple analytical formulas that accounts for the simultaneous variation of semi-major axis, inclination and right ascension of the ascending node.

The paper is structured as follows. After defining the target constellation, the paper introduces the approaches to define the deployment and launch sequences. It will then present the analytical formulas and the different transfer strategies followed by the multi-objective optimisation of the deployment and transfer of all the satellite in the constellation. It will be shown that some strategies are dominant and are to be preferred in the case analysed in this paper.

\section{Configuration of the Constellation}

The constellation considered in this study is a Walker Delta $56^{\circ}: 27 / 3 / 1$ constellation in MEO 11 In the notation $i: t / p / f$ used to describe Walker Delta constellation, $i$ represents the inclination of the orbit, $t$ the total number of satellites, $p$ the number of planes and $f$ is the relative spacing between satellites in adjacent planes. The semimajor axis of the orbits is $a_{M E O}=24200 \mathrm{~km}$ and the right ascension of the ascending node of the three planes are equally spaced of $120 \mathrm{deg}$. It is assumed that the only perturbation acting on the satellites is due to the second order zonal harmonic of the geopotential, J2. This perturbation causes the right ascension of the orbital plane to drift at a rate given by!

$$
\dot{\Omega}=-\frac{3}{2} n J_{2}\left(\frac{R_{\oplus}}{a_{M E O}}\right)^{2} \cos i_{M E O}
$$

where $n=\sqrt{\mu / a_{M E O}^{3}}$ is the mean motion of the satellite on its orbit, $\mu$ is the Earth's gravitational parameter, $R_{\oplus}$ is the Earth radius and $J_{2}=1.0826 \cdot 10^{-2}$ and $i_{M E O}=56 \mathrm{deg}$.

A graphical representation of the considered constellation is given in Figure 1, where the axis are in Earth- radii.

\section{Deployment Sequence}

In this section the method adopted to define the deployment sequence is described. The deployment sequence allows to define which satellite is allocated to which slot and the order with which these slots should be filled.

In this paper, the desired coverage strategy is the one that maximises the rate at which the constellation reaches its fully deployed coverage, i.e. that delays as much as possible eventual partial overlaps of coverage. To avoid dealing with a rather complex geometric problem involving the intersection of several spherical 


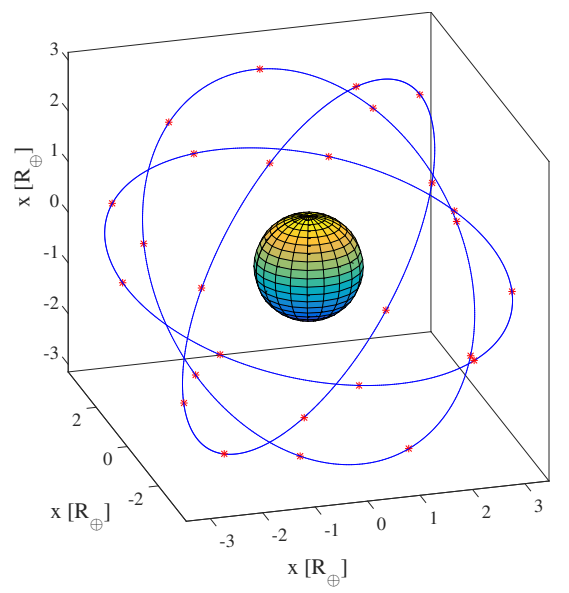

Figure 1. Walker Delta $56^{\circ}: 27 / 3 / 1$ constellation.

caps, the problem was tackled using an analogy: each satellite was considered an electrically charged particle that could be collocated in one of the available slots. By using this analogy, the desired coverage strategy translates into the strategy that minimises of the integral over time of the energy of the whole system, assuming that the time interval between the collocation of each subsequent satellite is constant.

A brute force analysis of all the possibilities for 27 satellites would require considering 27 ! combinations $(\approx$ $\left.10^{28}\right)$, so in order to solve the problem a greedy tree-search approach was used instead. With this approach, an initial position for the first satellite was chosen. Then, the integral over time of the energy resulting from the addition of one satellite was computed for all possible remaining positions, and all combinations with the same minimum were stored. With this approach, at each stage, a locally optimal choice is made, in an attempt to find the global optimum. This was repeated, stage by stage, for all promising combinations until all the satellites were collocated, and was also repeated for every possible choice for the initial satellite. This greedy approach does not guarantees to find the global optimal solution but provides good solutions in a reasonable amount of time. Due to the symmetries present in the problem, multiple equivalent optimal solutions are possible, but only one is considered in the following.

The optimal satellite deployment sequence obtained is shown in Figure 2 where the $\mathrm{x}$ axis indicates the number of the satellite and the y axis the plane (from 1 to 3 ) of arrival of the satellite.

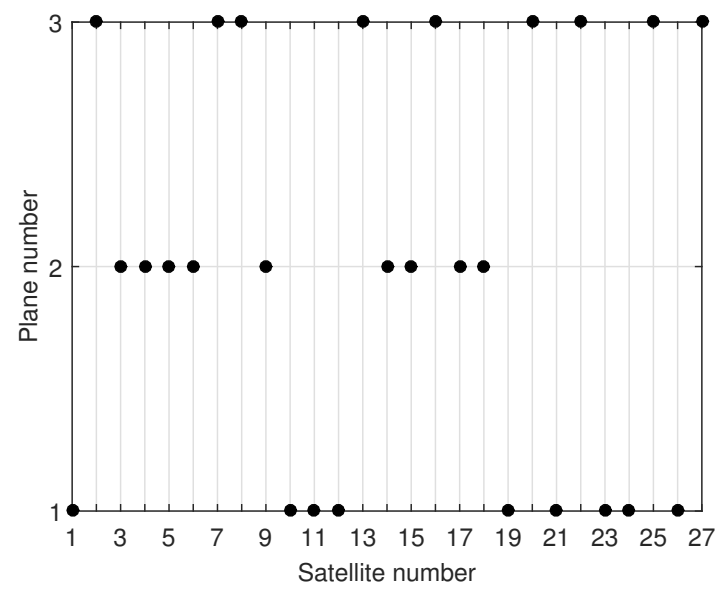

Figure 2. Representation of the optimal sequence of satellite deployment. 


\section{Launchers}

In this study two European launchers are considered: $\mathrm{Vega}^{3}$ and Ariane $5 \underline{4}^{4}$ For each launcher, models relating payload mass, target inclination and semimajor axis are developed from available data as simple second order bivariate polynomials. Figures 3 and 4 show the resulting surface for the two launchers.

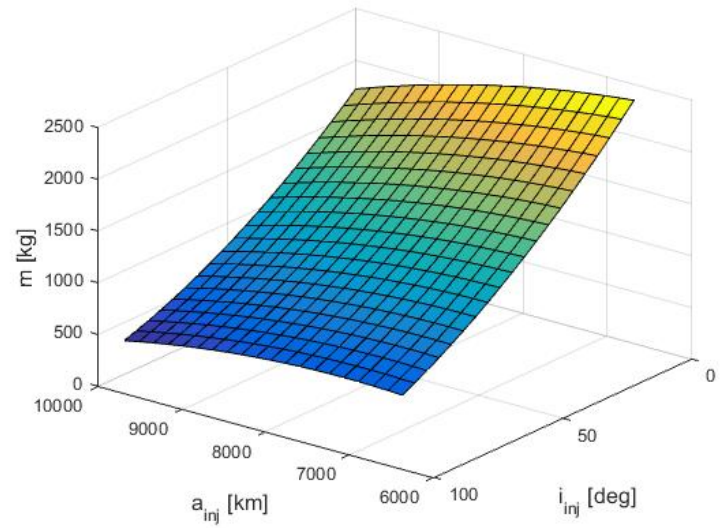

Figure 3. Relationship between mass and injection semimajor axis and inclination for Vega

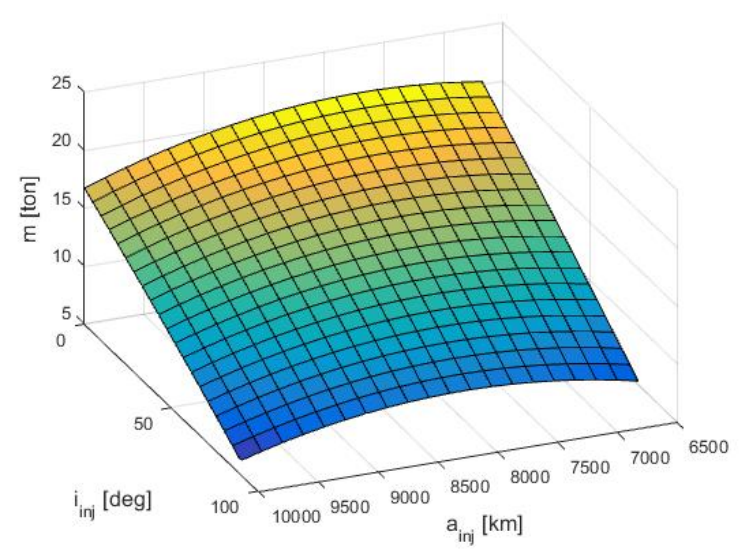

Figure 4. Relationship between mass and injection semimajor axis and inclination for Ariane

Due to fairing limitations, it is assumed that Vega can carry one satellite in orbit, while Ariane can carry four satellites of the constellation. With this assumption, and for a constellation of 27 satellites, 7 possible combinations of Ariane and Vega launches allow the deployment of the entire constellation. The total cost of the launches differ for the seven possibilities. Assuming a cost per launch of 32 million euros for Vega and 200 million euros for Ariane, the total cost reported in Table 1 can be obtained. The table also reports, for each combination, the total number of possible launch sequences.

Table 1. Combinations, launch sequences and total cost of Vega and Ariane launches for the deployment of a constellation of 27 satellites

\begin{tabular}{rrrrrrrr} 
& 1 & 2 & 3 & 4 & 5 & 6 & 7 \\
\hline Number Ariane launches & 0 & 1 & 2 & 3 & 4 & 5 & 6 \\
Number Vega launches & 27 & 23 & 19 & 15 & 11 & 7 & 3 \\
Possible launch sequences & 1 & 24 & 210 & 816 & 1365 & 792 & 84 \\
Total cost [M €] & 864 & 936 & 1008 & 1080 & 1152 & 1224 & 1296
\end{tabular}

Table 1 shows that two limit value exist for the cost of the launches. The minimum cost is obtained when all the launches are realised using Vega (option 1). The maximum cost is obtained when 6 launches are realised with Ariane and 3 with Vega (option 7). Assuming a rate of one launch per year, option 1 would require 27 years while option 7 would require 9 years, so there is a clear trade-off between total cost of the launches and total deployment time (which relates to a reduced pay-off). Due to the very large total number of possible launch sequences for the 7 deployment strategies (each of which would be followed by the solution of a bi-objective optimisation problem for the minimisation of the $\Delta V$ and the maximisation of pay-off), only a systematic study of the two limit cases was performed in the rest of the study.

\section{Low-Thrust Transfer}

The injection of the satellites on their final orbit can be obtained with a direct injection or with an indirect injection. In the direct injection case the launcher leaves the spacecraft on its final operational orbit. In the indirect injection case, the launcher leaves the spacecraft on a lower altitude orbit and the spacecraft moves to the final operation orbit using its propulsion system. In this work the indirect injection is considered and it is assumed that the spacecraft is equipped with low-thrust engine for the transfer from 
the injection to the final orbit.

Once the launchers leaves the satellite on the injection orbit, the low-thrust engine is operated to obtain the following variation of the orbital elements:

- $a_{i n j} \rightarrow a_{M E O}=a_{i n j}+\Delta a$

- $i_{\text {inj }} \rightarrow i_{M E O}=i_{\text {inj }}+\Delta i$

- $\Omega_{i n j} \rightarrow \Omega_{M E O}=\Omega_{i n j}+\Delta \Omega$

Four possible low-thrust strategies are considered to achieve these variations, simultaneously, in a given time of flight $T o F$. These strategies are presented in the following subsections.

A. Strategy 1: $\Delta \Omega_{J 2}+(\Delta a, \Delta i)$

In this case the transfer from injection to operational orbit is realised in two phases:

1. During the first phase the spacecraft waits on its initial injection orbit, where the effects of the drift of $\Omega$ due to J2 is higher (Equation 1). The low-thrust engine is off during this phase and the variation of $a$ and $i$ is zero. The time of flight associated to this phase is identified as $T o F_{1}^{S 1}$. The right ascension at the end of this phase is identified as $\Omega_{1 f}^{S 1}$. The variation in time of $\Omega$ is:

$$
\dot{\Omega}=-\frac{3}{2} \sqrt{\mu} J_{2} R_{\oplus}^{2} \cos i_{i n j} a_{i n j}^{-7 / 2}
$$

so that $T o F_{1}^{S 1}$ and $\Omega_{1 f}^{S 1}$ are linked by the following relationship:

$$
T o F_{1}^{S 1}=\frac{\Omega_{1 f}^{S 1}-\Omega_{i n j}}{\frac{3}{2} \sqrt{\mu} J_{2} R_{\oplus}^{2} \cos i_{i n j} a_{i n j}^{-7 / 2}}
$$

2. The second phase is realised switching on the low-thrust engine, during two thrust arcs per revolution. During this phase the semimajor axis and inclination change from their initial value to their final values. The variable drift due to $\mathrm{J} 2$ is such that at the end of the transfer the right ascension changes from $\Omega_{1 f}^{S 1}$ to $\Omega_{M E O}$. The time of flight of the second phase is $T o F_{2}^{S 1}$. The simultaneous variation of $a$ and $i$ in a given time of flight $T o F_{2}^{S 1}$ can be obtained with two tangential thrust arcs of semi-amplitude $\alpha$ and elevation angle $\beta$, centered at the nodal points of the orbit. $\beta$ has equal and opposite value on the two thrust arcs. The relevant equations for this transfer can be obtained from the Gauss equations for the variation in time of the semimajor axis and inclination of circular orbits with tangential thrust $[5$

$$
\begin{aligned}
& \frac{d a}{d t}=\frac{2 a^{2}}{h} f \cos \beta \\
& \frac{d i}{d t}=f \frac{a}{h} \cos u \sin \beta
\end{aligned}
$$

where $h$ is the angular momentum of the orbit, $f$ is the low-thrust acceleration and $u$ is the argument of the latitude.

The variation of $a$ and $i$ with the argument of the latitude $u$ of can be expressed as:

$$
\begin{aligned}
& \frac{d a}{d u}=\frac{d a}{d t} \frac{d t}{d u}=\frac{2 a^{3}}{\mu} f \cos \beta \\
& \frac{d i}{d u}=\frac{d i}{d t} \frac{d t}{d u}=\frac{f a^{2} \sin \beta \cos u}{\mu}
\end{aligned}
$$

The mean variation of $a$ over one orbital revolution can be obtained from:

$$
\begin{gathered}
\left(\frac{\overline{d a}}{d t}\right)=\frac{1}{T}\left[\int_{-\alpha}^{\alpha} \frac{d a}{d u}+\int_{\pi-\alpha}^{\pi+\alpha} \frac{d a}{d u}\right] \\
5 \text { of } 16
\end{gathered}
$$


to obtain:

$$
\frac{\overline{d i}}{d t}=\frac{2 f a \sin \beta \sin \alpha}{\pi \sqrt{\mu a}}
$$

Analogously, for the inclination:

$$
\frac{\overline{d i}}{d t}=\frac{2 f a \sin \beta \sin \alpha}{\pi \sqrt{\mu a}}
$$

In order for $a$ and $i$ to reach the final value simultaneously, the following equation is integrated from $a_{i n j}$ to $a_{M E O}$ and from $i_{i n j}$ to $i_{M E O}$ :

$$
\frac{d a}{d i}=\frac{2 a \alpha \cos \beta}{\sin \alpha \sin \beta}
$$

This results in:

$$
\tan \beta=\frac{2 \alpha\left(i_{M E O}-i_{i n j}\right)}{\sin \alpha \log \left(a_{M E O} / a_{i n j}\right)}
$$

The time of flight of the transfer can be expressed as a function of $\alpha$ and the initial and final orbital elements as:

$$
T o F_{2}^{S 1}=\frac{\pi}{2 f \alpha}\left(\sqrt{\frac{\mu}{a_{i n j}}}-\sqrt{\frac{\mu}{a_{M E O}}}\right) \sqrt{1+\frac{4 \alpha^{2}\left(i_{M E O}-i_{i n j}\right)^{2}}{\sin ^{2} \alpha \log ^{2}\left(a_{M E O} / a_{i n j}\right)}}
$$

By using the equation above it is possible to find $\alpha$ and therefore $\beta$ that allow to realise a variation of $a$ and $i$ in a given time of flight. The cost of the transfer can be computed analytically from:

$$
\Delta V=\frac{1}{\cos \beta}\left(\sqrt{\frac{\mu}{a_{i n j}}}-\sqrt{\frac{\mu}{a_{M E O}}}\right)
$$

During the variation of $a$ and $i$ the right ascension changes due to J2 and the variation of $a$ and $i$ with time. The value of $\Omega$ at the end of the second phase can be expressed as:

$$
\Omega_{2 f}^{S 1}=\Omega_{1 f}^{S 1}+\frac{k_{1}}{1+k_{2}^{2}} k_{3}
$$

where

$$
\begin{aligned}
& k_{1}=-\frac{3 \pi \mu J_{2} R^{2}}{4 a_{i n j}^{4} f \sin \beta \sin \alpha} \exp \left[\frac{4 \log \left(a_{M E O} / a_{i n j}\right) i_{i n j}}{\left(i_{M E O}-i_{i n j}\right)}\right] \\
& k_{2}=\frac{4 \log \left(a_{M E O} / a_{i n j}\right)}{\left(i_{M E O}-i_{i n j}\right)} \\
& k_{3}=\exp \left(k_{2} i_{M E O}\right)\left(k_{2} \cos i_{M E O}+\sin i_{M E O}\right)-\exp \left(k_{2} i_{i n j}\right)\left(k_{2} \cos i_{i n j}+\sin i_{i n j}\right)
\end{aligned}
$$

It has to be noted that Equations 14 become singular when $\left(i_{M E O}-i_{i n j}\right)$ is small. In the case $i_{M E O}=i_{i n j}$ an alternative non-singular formulation is available, but it is not presented here because the case $i_{i n j}=i_{M E O}$ is not considered in this work. 
If the combined transfer $\Delta \Omega_{J 2}+(\Delta a, \Delta i)$ has to be realised in a total time of flight $T o F_{t o t}$, the values of $T o F_{1}^{S 1}$ and $T o F_{2}^{S 1}$ can be computed solving the system of equations given by $\Omega_{2 f}^{S 1}=\Omega_{M E O}$ and:

$$
T o F_{1}^{S 1}+T o F_{1}^{S 2}=T o F_{t o t}
$$

and using Equations 3 and 13 . The possibility of realising the transfer by applying first $(\Delta a, \Delta i)$ and then $\Delta \Omega_{J 2}$ is not considered since the variation of $\Omega$ with $\mathrm{J} 2$ at high values of $a$, such as $a_{M E O}$, is limited (Equation 1). Figure 5 shows an example of transfer realised using this strategy, with time of flight of 550 days and low-thrust acceleration equal to $1.205 \cdot 10^{-4} \mathrm{~m} / \mathrm{s}^{2}$. The variation of semimajor axis is from 10000 to $24200 \mathrm{~km}$, the inclination changes from 51 to $56 \mathrm{deg}$ and the right ascension changes from 0 deg to 150 deg. The relevant parameter of the low-thrust control are $\alpha=49.13 \mathrm{deg}$ and $\beta=12.62 \mathrm{deg}$. The cost of the transfer is $\Delta V=2.31 \mathrm{~km} / \mathrm{s}$.
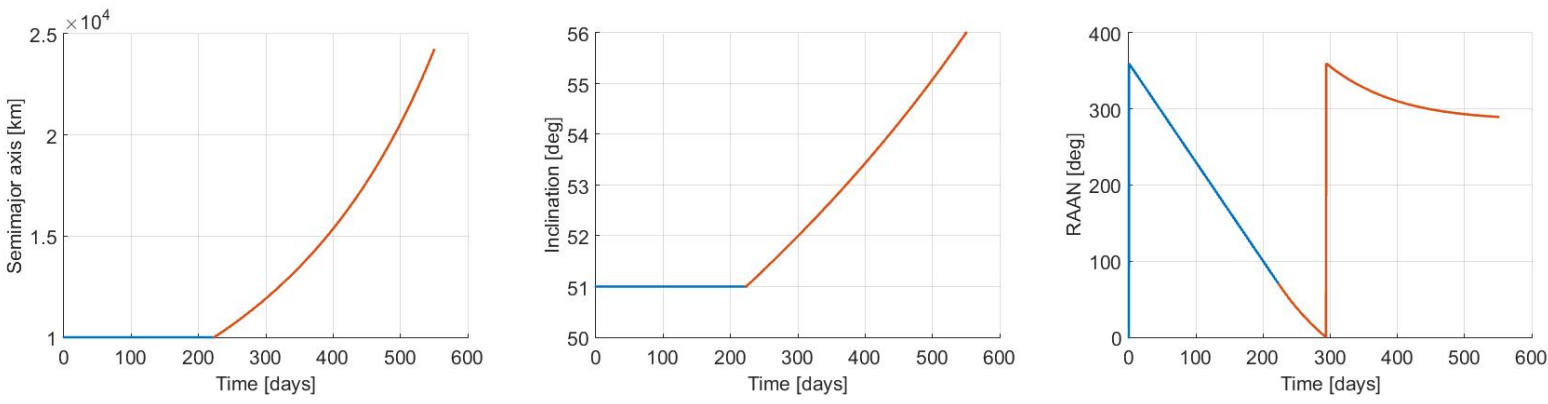

Figure 5. Variation of $a, i$ and $\Omega$ during low-thrust transfer with strategy 1.

B. Strategy 2: $(\Delta a, \Delta i)+\Delta \Omega_{\beta}$

In the second considered low-thrust strategy the transfer from injection to operational orbit is realised in two phases:

1. During the first phase $a$ and $i$ are changed from their initial values $a_{i n j}$ and $i_{i n j}$ to their final values in MEO using the same method used in phase 2 of Strategy 1, with low-thrust applied on two thrust arcs of semi-amplitude $\alpha_{1}$. This variation takes place in a time of flight $T o F_{1}^{S 2}$. The right ascension at the end of the first phase is computed from Equation 13 as:

$$
\Omega_{1 f}^{S 2}=\Omega_{i n j}+\frac{k_{1}}{1+k_{2}^{2}} k_{3}
$$

2. During the second phase $\Omega$ is changed using the low-thrust engine and out-of-plane thrust, with two thrust arcs of semi-amplitude $\alpha_{2}$ and elevation angle $\beta=90 \mathrm{deg}$ applied at the apsidal points of the orbit.

Using the Gauss equation for $\Omega$, the variation of $\Omega$ with time is given by:

$$
\frac{d \Omega}{d t}=f \frac{a \sin u \sin \beta}{h \sin i}
$$

The corresponding variation of $\Omega$ with the argument of the latitude is:

$$
\frac{d \Omega}{d u}=\frac{f a^{2} \sin u}{\sin i}
$$

The mean variation of $\Omega$ during one orbital revolution, due to both the out-of-plane thrust and J2, is:

$$
\frac{\overline{d \Omega}}{d t}=\frac{2 f \sin \alpha_{2}}{\pi \sin i} \sqrt{\frac{\mu}{a}}-\frac{3}{2} \sqrt{\mu} J_{2} R_{\oplus}^{2} \cos i a^{-7 / 2}
$$


If the variation of $\Omega$ has to be realised in a time of flight $T o F_{2}^{S 2}$, the semi-amplitude of the thrust arcs can be computed from:

$$
\sin \alpha_{2}=\frac{\frac{\Omega_{M E O}-\Omega_{1 f}^{S 2}}{T o F_{2}^{S 2}}+\frac{3}{2} \sqrt{\mu} J_{2} R_{\oplus}^{2} \cos i_{M E O} a_{M E O}^{-7 / 2}}{\frac{2 f}{\pi \sin i_{M E O}} \sqrt{\frac{\mu}{a_{M E O}}}}
$$

The cost associated to the variation of $\Omega$ can be computed analytically from:

$$
\Delta V=\sin i_{M E O} \sqrt{\frac{\mu}{a_{M E O}}} \frac{\alpha}{\sin \alpha}\left(\Omega_{M E O}-\Omega_{1 f}^{S 2}\right)
$$

When the combined transfer $(\Delta a, \Delta i)+\Delta \Omega_{\beta}$ has to be realised in a total time of flight $T o F_{t o t}$, the equations defined above, together with $T o F_{1}^{S 1}+T o F_{1}^{S 2}=T o F_{\text {tot }}$, do not provide a sufficient number of equations to solve the system and to find $\alpha_{1}$ and $\alpha_{2}$. It is possible however to define different arbitrary values of $T o F_{1}^{S 2}<T o F_{t o t}$ and compute the corresponding values of $\alpha_{1}, \alpha_{2}$ and $T o F_{2}^{S 2}$. In particular, it is possible to find a $T o F_{1}^{S 1}$ such that the $\Delta V$ of the transfer is the minimum possible value.

The possibility of realising the transfer by applying first $\Delta \Omega_{\beta}$ and then $(\Delta a, \Delta i)$ is not considered since the variation of $\Omega$ with J2 at low altitude is higher than the variation of $\Omega$ obtainable with the low-thrust engine.

An example of transfer realised with this strategy is shown in Figure 6. The initial and final orbital elements are those used for the previous example. The transfer is realised with $\Delta V=2.3045 \mathrm{~km} / \mathrm{s}$ and the parameter of the low-thrust control are $\alpha_{1}=32.70 \mathrm{deg}, \beta=11.78 \mathrm{deg}$ and $\alpha_{2}=21.77 \mathrm{deg}$.
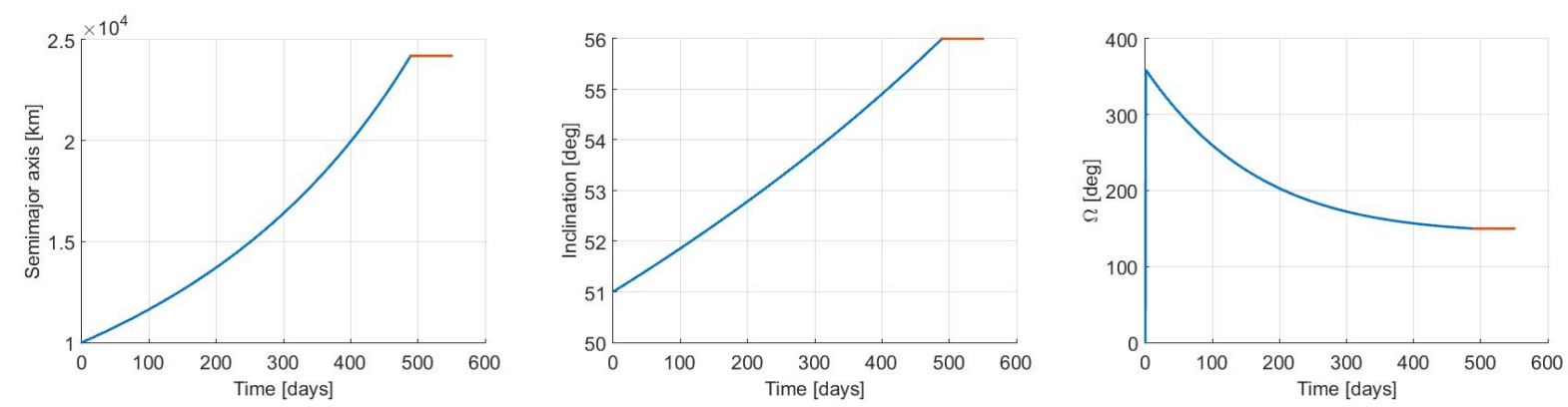

Figure 6. Variation of $a, i$ and $\Omega$ during low-thrust transfer with strategy 2.

\section{Strategy 3: $(\Delta a, \Delta \Omega)_{N o W a i t}+\Delta i$}

The low-thrust transfer using strategy 3 is realised in two phases:

1. During the first phase a tangential thrust with $\beta=0$ is used to increase the semimajor axis from $a_{i n j}$ to $a_{M E O}$. The engine is on during two thrust arcs per revolution, of semi-amplitude $\alpha_{1}$.

The time of flight is given by:

$$
T o F_{1}^{S 1}=\frac{\pi}{2 f \alpha_{1}}\left(\sqrt{\frac{\mu}{a_{i n j}}}-\sqrt{\frac{\mu}{a_{M E O}}}\right)
$$

The cost of this phase can be computed analytically from:

$$
\Delta V=\sqrt{\frac{\mu}{a_{i n j}}}-\sqrt{\frac{\mu}{a_{M E O}}}
$$


The right ascension at the end of the transfer can be obtained by integrating $d \Omega / d a$ obtained from the equations for $d \Omega / d t$ and $d a / d t$. This results in:

$$
\Omega_{1 f}^{S 3}=\Omega_{i n j}+\frac{3}{32} \frac{\mu J_{2} R_{\oplus}^{2} \cos i_{i n j}}{f \alpha_{1}}\left(\frac{1}{a_{M E O}^{4}}-\frac{1}{a_{i n j}^{4}}\right)
$$

2. During the second phase the thrust is applied during two thrust arcs per revolution, of semi-amplitude $\alpha_{2}$, with elevation $\beta=90 \mathrm{deg}$, to change the inclination from $i_{i n j}$ to $i_{M E O}$.

The time required can be computed from:

$$
T_{o F}^{S 2}=\frac{i_{M E O}-i_{i n j}}{\frac{2 f}{\pi} \sqrt{\frac{\mu}{a}} \sin \alpha_{2}}
$$

The cost of this phase is:

$$
\Delta V=\frac{\alpha_{2}}{\sin \alpha_{2}} \sqrt{\frac{\mu}{a_{M E O}}}\left(i_{M E O}-i_{i n j}\right)
$$

The variation of right ascension during this phase can be obtained from Equation 13 and subsection A as particular case in which the variation of semimajor axis is zero:

$$
\Omega_{2 f}^{S 3}=\Omega_{1 f}^{S 3}+\frac{3}{4} \frac{\pi \mu J_{2} R_{\oplus}^{2}}{f a_{M E O}^{4} \sin \alpha_{2}}\left(\sin i_{i n j}-\sin i_{M E O}\right)
$$

The above equations can be used to solve the problem in which the entire transfer has to be realised in a given time of flight. In particular the equations to satisfy are:

$$
\begin{aligned}
& T o F_{1}^{S 3}+T o F_{2}^{S 3}=T o F_{t o t} \\
& \Omega_{2 f}^{S 3}=\Omega_{M E O}
\end{aligned}
$$

The possibility of realising the transfer by applying first $\Delta i$ and then $(\Delta a, \Delta \Omega)$ is not considered since the cost of the variation of $i$ at low altitude is higher.

An example of transfer realised with this strategy is shown in Figure 7. The transfer is realised with $\Delta V=2.6201 \mathrm{~km} / \mathrm{s}, \alpha_{1}=33.68 \mathrm{deg}$ and $\alpha_{2}=30.31 \mathrm{deg}$.
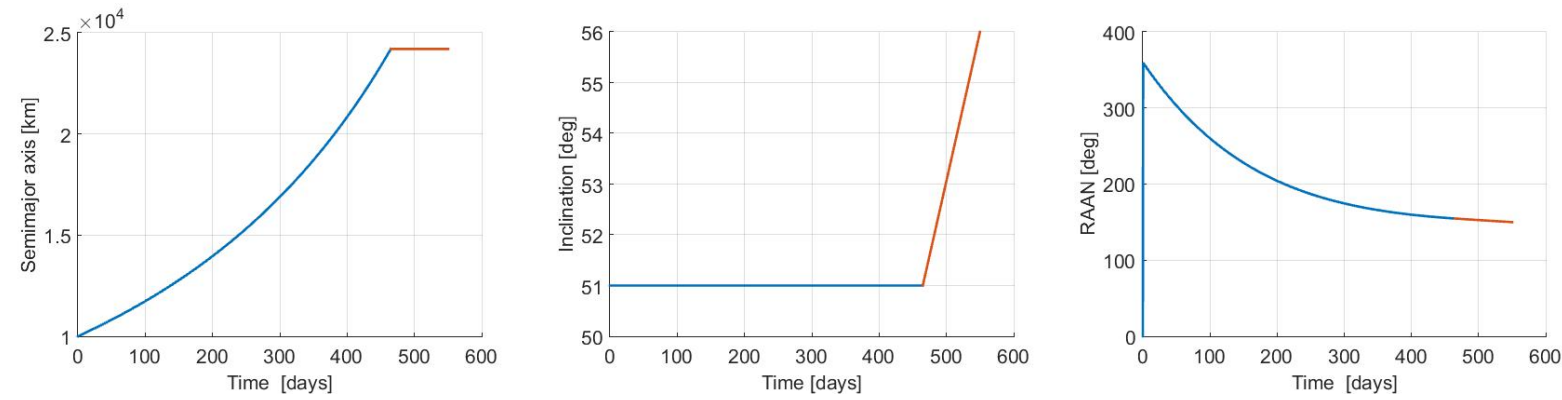

Figure 7. Variation of $a, i$ and $\Omega$ during low-thrust transfer with strategy 3.

D. Strategy 4: $(\Delta a, \Delta \Omega)_{W a i t}+\Delta i$

This strategy is analogous to strategy 3 presented above. The difference is in the introduction of a waiting time $T_{\text {wait }}^{S 4}$, during phase 1 , when the engine is off and the drift of $\Omega$ due to $\mathrm{J} 2$ can be exploited. The first of Equations 28 is modified as:

$$
T_{w a i t}^{S 4}+T o F_{1}^{S 4}+T o F_{2}^{S 4}=T o F_{t o t}
$$


and Equation 27 is now expressed as:

$$
\Omega_{1 f}^{S 4}=\Omega_{i n j}+\frac{3}{32} \frac{\mu J_{2} R_{\oplus}^{2} \cos i_{i n j}}{f \alpha_{1}}\left(\frac{1}{a_{M E O}^{4}}-\frac{1}{a_{i n j}^{4}}\right)-\dot{\Omega} T_{w a i t}^{S 4}
$$

In this case, as for strategy 2, the number of relevant equations is not sufficient to solve the problem when the transfer has to be realised in a total time of flight $T o F_{t o t}$. A value of $T o F_{1}^{S 4}<T o F_{\text {tot }}$ exist however for which the $\Delta V$ of the transfer is minimum.

An example of transfer realised with this strategy is shown in Figure 8 . The blue lines represent the waiting time on the initial orbit. The transfer is realised with $\Delta V=2.6104 \mathrm{~km} / \mathrm{s}$ and $\alpha_{1}=85.81 \mathrm{deg}$ and $\alpha_{2}=8.22 \mathrm{deg}$.
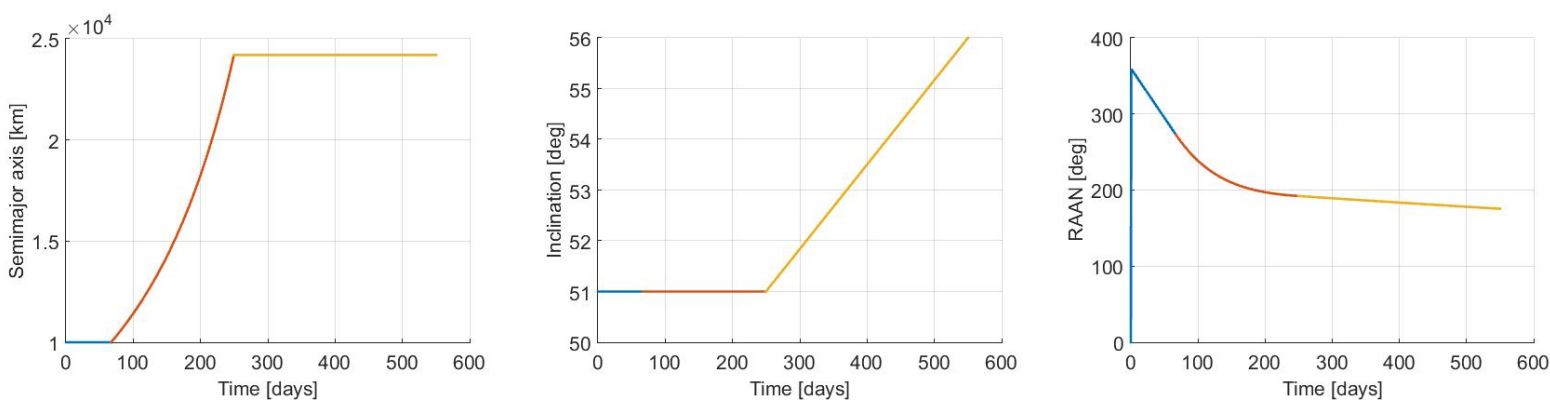

Figure 8. Variation of $a, i$ and $\Omega$ during low-thrust transfer with strategy 4.

\section{E. Low-thrust strategies comparison}

Figure 9 shows the $\Delta V$ required to realise the transfer defined in the previous example, for different values of the times of flight and using the four strategies defined in the previous subsection. For strategies 2 and 4 , where a unique solution does not exist, the plotted solutions are the ones corresponding to the values of $T o F_{1}$ and $T o F_{2}$ providing the lower value of the $\Delta V$ for that transfer.

For the multi-objective optimisation of constellation deployment strategy 1 is considered. This gives lower $\Delta V$ than strategy 3 and 4 and, compared to strategy 2 , does not require out-of-plane maneuvers to change the right ascension of the ascending node.

The discontinuity in the curve relative to strategy 1 at $T o F$ of approximately 600 days is due to a jump of the variation of $\Omega$, during the first phase, from less than $2 \pi$ to a variation of more than $2 \pi$, that is a jump from $\left|\Omega_{1 f}^{S 1}-\Omega_{i n j}\right|<2 \pi$ to $\left|\Omega_{1 f}^{S 1}-\Omega_{i n j}\right|>2 \pi$. This causes the time of flight available for the second phase to be reduced of an amount equal to $2 \pi / \dot{\Omega}$, thus increasing the total $\Delta V$. It has to be noted that a variation of more than $2 \pi$ during the first phase of strategy 1 might be necessary in order to reach simultaneously the final orbital elements at the end of the transfer.

\section{Multi-Objective Deployment Optimisation}

The objectives of the optimisation of the constellation deployment are the minimisation of the maximum $\Delta V$ of all the low-thrust transfer, the maximisation of the profit of the constellation and the minimisation of the cost of the launches. As regards the cost of the launches, the two extreme cases in Table 1 are considered. The objectives considered in the following are therefore:

- Minimisation of the maximum $\Delta V$ of the low-thrust transfers

- Maximisation of the profit obtained from the deployment of the constellation. Each satellite is assumed to generate profit from the moment it reaches its final orbit, up to an an end date defined as 5 years after the last launch. The adimensional profit rate considered in this work is of one unit per day.

The multi-objective problem is solved using the memetic multi-objective optimiser Multi Agent Collaborative Search (MACS2)! ${ }^{[}$Multi-Agent Collaborative Search is a meta-heuristic that combines local and global search heuristics. A set of agents is endowed with a list of possible actions that can involve other 


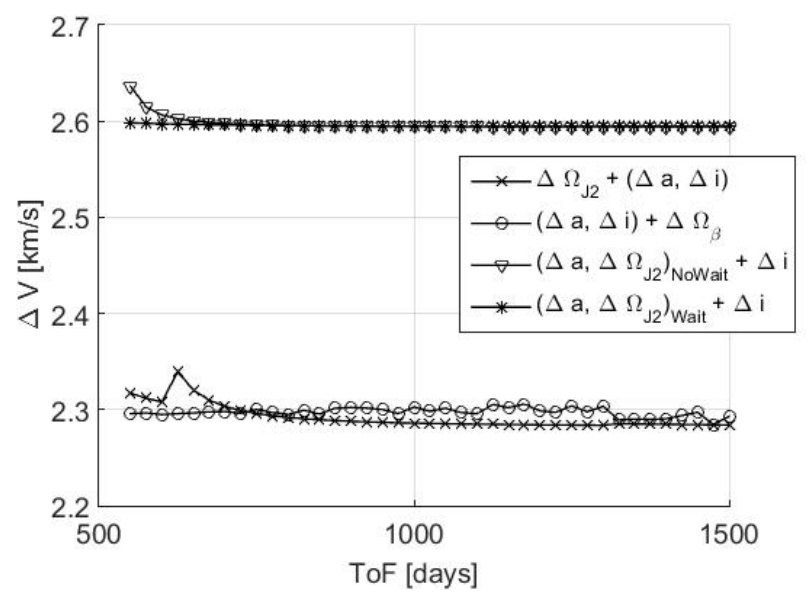

Figure 9. $\Delta V$ of the low-thrust transfer for different time of flights and low-thrust strategies.

agents or simply collect information on a neighborhood of each agent. In MACS2 the idea of search directions was introduced in the logic of the agents, which could select new candidate solutions according to either dominance or Tchebycheff scalarisation. All solutions that are non-dominated or satisfy Tchebycheff scalarisation criterion are stored in a global archive that contains the best approximation of the Pareto front. Therefore, at each iteration of MACS2, archive maintains the set of locally Pareto optimal solutions, while the populations of agents explore the parameter space in search for improvements. MACS2 was shown to be very effective compared to more traditional multi-objective optimisers at providing a good balance of convergence and spreading of the solutions.

The vector of optimisable parameters, that is handled by MACS2, includes, for each satellite launched:

- Semimajor axis of the injection orbit, $a_{i n j}$

- Inclination of the injection orbit, $i_{i n j}$

- Right ascension of the ascending node of the injection orbit, $\Omega_{i n j}$

- Time of flight from the injection to the operational orbit, ToF

When four satellites are launched with Ariane, the time of flights of the four satellites are constrained such that the sequence of deployment defined in Section III is still satisfied. The final semimajor axis and inclination of the arrival orbit are $a_{M E O}$ and $i_{M E O}$. The right ascension of the arrival orbits is computed from Equation 1. based on the arrival time of the satellite on the selected orbit. The boundaries for the parameters to optimise are:

$$
\begin{aligned}
& 6878 \mathrm{~km} \leq a_{i n j} \leq 9378 \mathrm{~km} \\
& 0 \mathrm{deg} \leq i_{i n j} \leq 50 \mathrm{deg} \\
& 0 \operatorname{deg} \leq \Omega_{i n j} \leq 360 \mathrm{deg} \\
& 300 \text { days } \leq \text { ToF } \leq 1500 \text { days }
\end{aligned}
$$

The selected values of admissible $a_{i n j}$ have been chosen in order to avoid regions where the effect of the drag is not negligible $(a<6878 \mathrm{~km})$ and regions where the drift of $\Omega$ due to $J_{2}$ is not very significant $(a>9378 \mathrm{~km})$. Likewise the range of inclinations is restricted to exploit natural dynamics for the change of $\Omega$.

The considered value of the acceleration for the low-thrust transfer is $1.20510^{-4} \mathrm{~m} / \mathrm{s}^{2}$.

\section{Results}

This section presents the results obtained for the two selected cases in Table 1 maximum launch cost with minimum launch time, minimum launch cost with maximum launch time. 


\section{A. Maximum launch cost with minimum launch time}

Option 7 in Table 1 identifies the solution with minimum launch times (9 years, in the assumption of 1 launch per year) but maximum cost for the launches (1296 million euros). The total cost of the 9 launches is fixed but different combinations of sequences of Ariane and Vega launches exist for that cost. In particular, 84 possible combinations of launches can be identified; some of these combinations are presented in Table 2 . where V stands for launch with Vega and A for launch with Ariane.

Table 2. Possible combinations of Ariane and Vega launches for solution with deployment of the constellation in 9 launches

\begin{tabular}{r|ccccccccc}
\hline Comb. & 1 & 2 & 3 & 4 & 5 & 6 & 7 & 8 & 9 \\
\hline 1 & V & V & V & A & A & A & A & A & A \\
2 & V & V & A & V & A & A & A & A & A \\
$\vdots$ & & & & & & & & & \\
78 & A & A & A & A & V & A & V & V & A \\
$\vdots$ & & & & & & & & & \\
81 & A & A & A & A & A & V & V & V & A \\
82 & A & A & A & A & A & V & V & A & V \\
83 & A & A & A & A & A & V & A & V & V \\
84 & A & A & A & A & A & A & V & V & V
\end{tabular}

Each combination defined in Table 2 generates a Pareto set in the plane $\Delta V_{\max }$-Profit. The 84 Pareto sets obtained are shown in Figure 10, with different color for each combination. A single Pareto set can be obtained by considering the non-dominated solution of the 84 combinations. This is shown in Figure 11 . along with a number identifying the sequence of Vega and Ariane launchers (Table 2 ).

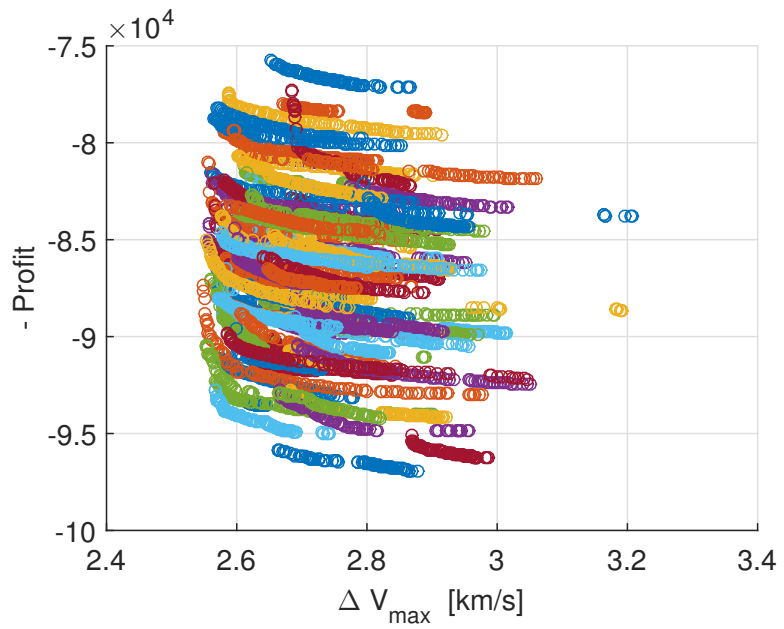

Figure 10. Pareto sets of the 84 combinations of launches corresponding to option 7 in Table 1 .

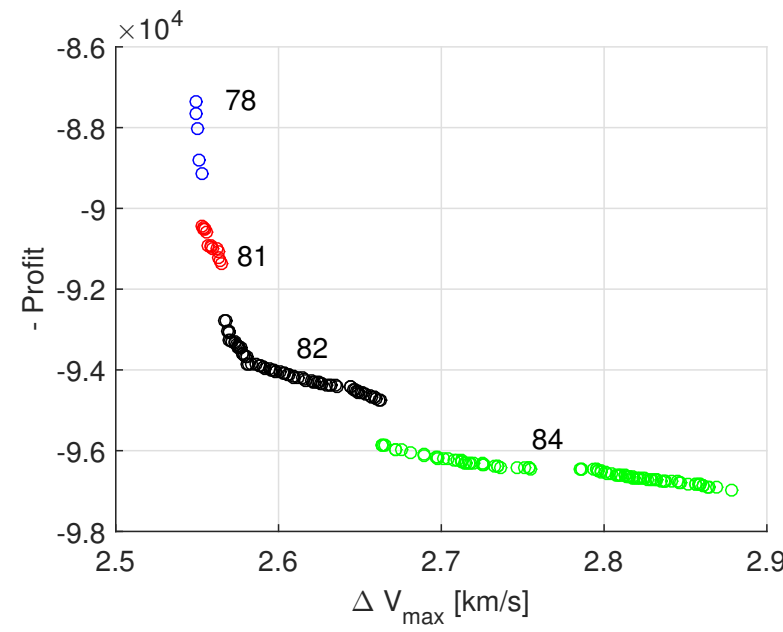

Figure 11. Non-dominated solutions resulting from the combinations of the $\mathbf{8 4}$ Pareto sets in Figure 10

Results in Figure 11, show that only four combinations of launches give non-dominated results. These are combinations $78,81,82$ and 84 . The sequence of launchers for these four cases can be found in Table 2. The solutions of combination 84 are characterised by higher profit and higher maximum $\Delta V$ while the solutions of combination 78 are characterised by lower profit and lower maximum $\Delta V$.

Details of the two extreme solutions of the Pareto set are presented in Tables 3 and 4 . The table shows the number of the plane where each satellite is launched, the year of the launch, the name of the launcher, the time of flight and $\Delta V$ associated to the low-thrust transfer, the parameters of the injection orbit and 
the right ascension of the final orbit.

The times of flight in Table 3 are averagely higher than the times of flight in Table 4 Higher times of flight results in lower $\Delta V$ but also lower pay-off from the constellation, since the final time of full disposal of the constellation is shifted in time.

The optimal injection orbits are in both cases characterised by a value of the inclination $i_{i n j}$ close to the upper limit of $50 \mathrm{deg}$. This is due to the fact that changes of inclination are expensive in term of $\Delta V$ and require long times of flight and are therefore penalised both in term of $\Delta V_{\max }$ and profit.

Table 3. Solution with minimum maximum $\Delta V$ and lower profit (combination of launches 78)

\begin{tabular}{|c|c|c|c|c|c|c|c|c|}
\hline Plane ID & Year & Launcher & ToF [days] & $\Delta V[\mathrm{~km} / \mathrm{s}]$ & $a_{i n j}[\mathrm{~km}]$ & $i_{i n j}[\mathrm{deg}]$ & $\Omega_{i n j}[\mathrm{deg}]$ & $\Omega_{M E O}[\mathrm{deg}]$ \\
\hline 1 & 1 & Ariane & 624.47 & 2.53 & 9378.00 & 49.93 & 0.00 & 327.30 \\
\hline 3 & 1 & Ariane & 625.47 & 2.54 & 9378.00 & 49.93 & 0.00 & 207.25 \\
\hline 2 & 1 & Ariane & 637.20 & 2.55 & 9378.00 & 49.93 & 0.00 & 86.63 \\
\hline 2 & 1 & Ariane & 638.20 & 2.55 & 9378.00 & 49.93 & 0.00 & 86.58 \\
\hline 2 & 2 & Ariane & 519.19 & 2.55 & 9378.00 & 50.00 & 157.03 & 73.68 \\
\hline 2 & 2 & Ariane & 520.19 & 2.55 & 9378.00 & 50.00 & 157.03 & 73.63 \\
\hline 3 & 2 & Ariane & 521.19 & 2.53 & 9378.00 & 50.00 & 157.03 & 193.58 \\
\hline 3 & 2 & Ariane & 522.19 & 2.53 & 9378.00 & 50.00 & 157.03 & 193.53 \\
\hline 2 & 3 & Ariane & 484.95 & 2.54 & 9378.00 & 50.00 & 0.00 & 56.35 \\
\hline 1 & 3 & Ariane & 500.58 & 2.55 & 9378.00 & 50.00 & 0.00 & 295.53 \\
\hline 1 & 3 & Ariane & 501.58 & 2.55 & 9378.00 & 50.00 & 0.00 & 295.48 \\
\hline 1 & 3 & Ariane & 502.58 & 2.55 & 9378.00 & 50.00 & 0.00 & 295.43 \\
\hline 3 & 4 & Ariane & 838.05 & 2.53 & 9377.98 & 50.00 & 70.56 & 138.73 \\
\hline 2 & 4 & Ariane & 839.09 & 2.53 & 9377.98 & 50.00 & 70.56 & 18.67 \\
\hline 2 & 4 & Ariane & 840.09 & 2.53 & 9377.98 & 50.00 & 70.56 & 18.62 \\
\hline 3 & 4 & Ariane & 841.09 & 2.53 & 9377.98 & 50.00 & 70.56 & 138.57 \\
\hline 2 & 5 & Vega & 448.68 & 2.55 & 9378.00 & 50.00 & 0.00 & 19.99 \\
\hline 2 & 6 & Ariane & 655.91 & 2.54 & 9378.00 & 49.92 & 226.14 & 350.01 \\
\hline 1 & 6 & Ariane & 656.91 & 2.53 & 9378.00 & 49.92 & 226.14 & 229.96 \\
\hline 3 & 6 & Ariane & 657.91 & 2.53 & 9378.00 & 49.92 & 226.14 & 109.91 \\
\hline 1 & 6 & Ariane & 658.91 & 2.53 & 9378.00 & 49.92 & 226.14 & 229.86 \\
\hline 3 & 7 & Vega & 412.90 & 2.55 & 9378.00 & 50.00 & 21.06 & 103.61 \\
\hline 1 & 8 & Vega & 579.87 & 2.55 & 9378.00 & 49.99 & 7.71 & 195.74 \\
\hline 1 & 9 & Ariane & 677.39 & 2.53 & 9374.53 & 49.96 & 199.03 & 171.50 \\
\hline 3 & 9 & Ariane & 678.40 & 2.53 & 9374.53 & 49.96 & 199.03 & 51.45 \\
\hline 1 & 9 & Ariane & 679.40 & 2.53 & 9374.53 & 49.96 & 199.03 & 171.40 \\
\hline 3 & 9 & Ariane & 680.40 & 2.53 & 9374.53 & 49.96 & 199.03 & 51.35 \\
\hline
\end{tabular}

\section{B. Minimum launch cost with maximum launch time}

Option 1 in Table 1 identifies the solution with maximum launch times (27 years, under the assumption of 1 launch per year) but minimum total cost for the launches (864 million euros).

In this case only one combination exist for the 27 launches with Vega. The resulting Pareto front is shown in Figure 12 .

The higher profit with respect to the case of maximum launch cost and minimum launch time is due to the fact that the profit is computed based on the time required for the full deployment of the constellation. In this case the deployment takes 27 years, plus the time of flight of the last satellite, while it was 9 years in the previous case. Table 5 shows details of the solution with higher maximum $\Delta V$ and higher profit. 
Table 4. Solution with higher maximum $\Delta V$ and higher profit (combination of launches 84)

\begin{tabular}{|c|c|c|c|c|c|c|c|c|}
\hline Plane ID & Year & Launcher & ToF [days] & $\Delta V[\mathrm{~km} / \mathrm{s}]$ & $a_{i n j}[\mathrm{~km}]$ & $i_{i n j}[\mathrm{deg}]$ & $\Omega_{i n j}[\mathrm{deg}]$ & $\Omega_{M E O}[\mathrm{deg}]$ \\
\hline 1 & 1 & Ariane & 434.47 & 2.87 & 8623.69 & 50.00 & 146.72 & 337.25 \\
\hline 3 & 1 & Ariane & 435.47 & 2.82 & 8623.69 & 50.00 & 146.72 & 217.19 \\
\hline 2 & 1 & Ariane & 436.47 & 2.83 & 8623.69 & 50.00 & 146.72 & 97.14 \\
\hline 2 & 1 & Ariane & 437.47 & 2.83 & 8623.69 & 50.00 & 146.72 & 97.09 \\
\hline 2 & 2 & Ariane & 405.06 & 2.85 & 8719.53 & 49.68 & 177.65 & 79.66 \\
\hline 2 & 2 & Ariane & 406.06 & 2.85 & 8719.53 & 49.68 & 177.65 & 79.61 \\
\hline 3 & 2 & Ariane & 407.06 & 2.80 & 8719.53 & 49.68 & 177.65 & 199.56 \\
\hline 3 & 2 & Ariane & 408.06 & 2.80 & 8719.53 & 49.68 & 177.65 & 199.50 \\
\hline 2 & 3 & Ariane & 452.77 & 2.84 & 8594.14 & 50.00 & 92.03 & 58.03 \\
\hline 1 & 3 & Ariane & 453.77 & 2.86 & 8594.14 & 50.00 & 92.03 & 297.98 \\
\hline 1 & 3 & Ariane & 454.77 & 2.86 & 8594.14 & 50.00 & 92.03 & 297.93 \\
\hline 1 & 3 & Ariane & 455.77 & 2.86 & 8594.14 & 50.00 & 92.03 & 297.88 \\
\hline 3 & 4 & Ariane & 420.57 & 2.83 & 8685.03 & 49.46 & 178.91 & 160.59 \\
\hline 2 & 4 & Ariane & 421.57 & 2.88 & 8685.03 & 49.46 & 178.91 & 40.54 \\
\hline 2 & 4 & Ariane & 422.57 & 2.87 & 8685.03 & 49.46 & 178.91 & 40.49 \\
\hline 3 & 4 & Ariane & 423.57 & 2.82 & 8685.03 & 49.46 & 178.91 & 160.44 \\
\hline 2 & 5 & Ariane & 501.19 & 2.84 & 9294.10 & 46.04 & 221.98 & 17.24 \\
\hline 2 & 5 & Ariane & 502.19 & 2.84 & 9294.10 & 46.04 & 221.98 & 17.19 \\
\hline 1 & 5 & Ariane & 503.19 & 2.69 & 9294.10 & 46.04 & 221.98 & 257.14 \\
\hline 3 & 5 & Ariane & 504.19 & 2.73 & 9294.10 & 46.04 & 221.98 & 137.09 \\
\hline 1 & 6 & Ariane & 388.98 & 2.82 & 8666.91 & 49.99 & 189.51 & 243.99 \\
\hline 3 & 6 & Ariane & 389.98 & 2.86 & 8666.91 & 49.99 & 189.51 & 123.94 \\
\hline 1 & 6 & Ariane & 390.98 & 2.81 & 8666.91 & 49.99 & 189.51 & 243.89 \\
\hline 1 & 6 & Ariane & 391.98 & 2.81 & 8666.91 & 49.99 & 189.51 & 243.83 \\
\hline 3 & 7 & Vega & 363.79 & 2.83 & 8728.84 & 49.95 & 104.34 & 106.18 \\
\hline 1 & 8 & Vega & 362.44 & 2.88 & 8821.09 & 48.11 & 186.58 & 207.13 \\
\hline 3 & 9 & Vega & 333.83 & 2.84 & 8787.84 & 49.48 & 10.25 & 69.50 \\
\hline
\end{tabular}

The higher values of $\Delta V$ are due to the limited launch capabilities of Vega with respect to Ariane. The inclination of the injection orbit is indeed lower than in Table 3 and 4 .

\section{Conclusions}

The paper presented the study of the deployment of a constellation of 27 satellites on 3 planes in MEO using low-thrust propulsion and two possible launchers, Ariane and Vega. A sub-optimal but effective sequence was defined for the launches of the satellite, to allocate each satellite to a specific slot of the constellation. Two launch options (maximum launch time with minimum launch cost and minimum launch time with maximum launch cost) were studied. The transfer from injection to operation orbit was realised with low-thrust trajectories that exploit the natural perturbations due to the Earth's gravitational potential. Pareto sets were generated to show the relationship between the pay-off of the constellation and the the mass of propellant required for the most expensive low-thrust transfer from injection to operational orbit. Results showed that only four combinations of sequence of launches constitute the points of the non-dominated Pareto set for the case of minimum launch time with maximum launch cost. In this case the launch of the constellation can be realised in 9 years, with increase in the pay-off that could be obtained with small variations of the maximum $\Delta V$. The extreme case of maximum launch time (27 years) with minimum launch 


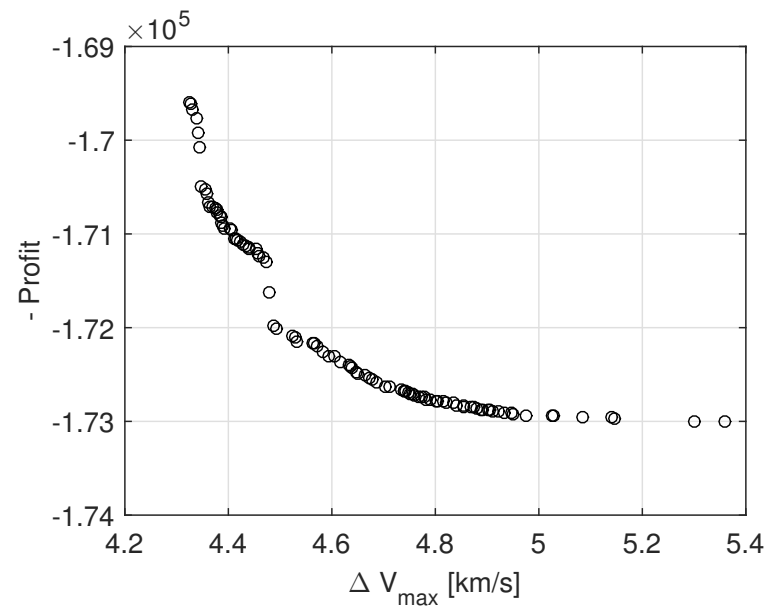

Figure 12. Pareto set corresponding to option 1 in Table 1 .

cost was also studied. In this case the sequence of launches is fixed and only one Pareto set exists.

Future study will consider the possibility of realising the low-thrust transfer with an out-of-plane manuever to change the right ascension of the orbit and will extend the study to all the possible range of costs of the launches options.

\section{Acknowledgments}

This research was partially funded by Airbus Defence and Space and partially by the ESA NPI programme. The authors would like to thank Mr Stephen Kemble, at Airbus DS, for his support and advice. The authors would like to thank the Institute of Engineering and Technology for the IET Travel Award to attend the Space 2016 Forum and present this work.

\section{References}

\footnotetext{
${ }^{1}$ Fortescue, P., Stark, J., Swinerd, G., Spacecraft System Engineering, Wiley, 2011

${ }^{2}$ Vallado, D., Fundamentals of Astrodynamics and Applications, Space Technology Library, 2007

${ }^{3}$ http://www.arianespace.com/wp-content/uploads/2015/09/Vega-Users-Manual_Issue-04_April-2014.pdf, Accessed May

${ }^{4}$ http://www.arianespace.com/wp-content/uploads/2015/09/Ariane5_users_manual_Issue5_July2011.pdf, Accessed May

${ }^{5}$ Battin, R. H, An introduction to the mathematics and methods of astrodynamics, AIAA, 1999.

${ }^{6}$ Ricciardi, L., Vasile, M., Improved archiving and search strategies for Multi Agent Collaborative Search, International Conference on Evolutionary and Deterministic Methods for Design, Optimization and Control with Applications to Industrial and Societal Problems, EUROGEN-2015, Glasgow, 14-16 September 2016
} 2016. 2016. 
Table 5. Solution with higher maximum $\Delta V$ and higher profit for launches with Vega only

\begin{tabular}{rrrrrrrrr} 
Plane ID & Year & Launcher & ToF [days $]$ & $\Delta V[\mathrm{~km} / \mathrm{s}]$ & $a_{\text {inj }}[\mathrm{km}]$ & $i_{\text {inj }}[\mathrm{deg}]$ & $\Omega_{\text {inj }}[\mathrm{deg}]$ & $\Omega_{M E O}[\mathrm{deg}]$ \\
\hline 1 & 1 & Vega & 479.96 & 4.10 & 8056.69 & 35.19 & 202.69 & 334.87 \\
3 & 2 & Vega & 538.48 & 5.10 & 7674.50 & 27.23 & 158.37 & 192.67 \\
2 & 3 & Vega & 434.60 & 4.21 & 7922.37 & 35.85 & 196.60 & 58.99 \\
2 & 4 & Vega & 534.96 & 4.49 & 8091.04 & 33.18 & 118.76 & 34.60 \\
2 & 5 & Vega & 461.75 & 3.75 & 8338.37 & 38.84 & 245.55 & 19.31 \\
2 & 6 & Vega & 623.39 & 4.79 & 8101.14 & 26.79 & 149.88 & 351.72 \\
3 & 7 & Vega & 618.04 & 4.95 & 8154.60 & 26.34 & 254.89 & 92.87 \\
3 & 8 & Vega & 592.09 & 5.16 & 8139.59 & 26.36 & 190.83 & 75.10 \\
2 & 9 & Vega & 551.55 & 4.65 & 8116.09 & 28.58 & 247.00 & 298.09 \\
1 & 10 & Vega & 562.23 & 4.84 & 7893.93 & 28.91 & 244.28 & 158.41 \\
1 & 11 & Vega & 505.64 & 4.95 & 7458.27 & 30.74 & 141.15 & 142.24 \\
1 & 12 & Vega & 385.42 & 3.59 & 7822.33 & 42.63 & 173.26 & 129.41 \\
3 & 13 & Vega & 515.57 & 4.81 & 8554.87 & 28.90 & 192.58 & 343.47 \\
2 & 14 & Vega & 462.22 & 4.01 & 8039.53 & 37.85 & 121.40 & 207.13 \\
2 & 15 & Vega & 509.53 & 4.88 & 8045.47 & 29.79 & 103.71 & 185.53 \\
3 & 16 & Vega & 556.41 & 4.12 & 8920.56 & 29.71 & 106.02 & 283.95 \\
2 & 17 & Vega & 573.93 & 4.95 & 8221.12 & 26.17 & 127.12 & 143.90 \\
2 & 18 & Vega & 576.23 & 4.85 & 8092.05 & 28.47 & 224.02 & 124.65 \\
1 & 19 & Vega & 471.27 & 4.57 & 8154.85 & 32.28 & 176.69 & 351.02 \\
3 & 20 & Vega & 550.67 & 4.70 & 8592.51 & 29.14 & 156.64 & 207.74 \\
1 & 21 & Vega & 551.40 & 4.94 & 8370.21 & 25.38 & 160.31 & 308.57 \\
3 & 22 & Vega & 639.89 & 4.83 & 8471.34 & 24.20 & 208.49 & 164.81 \\
1 & 23 & Vega & 573.90 & 4.95 & 7973.60 & 25.48 & 207.63 & 269.14 \\
1 & 24 & Vega & 508.33 & 4.31 & 8094.43 & 32.24 & 134.14 & 253.44 \\
3 & 25 & Vega & 609.78 & 5.36 & 8417.44 & 22.98 & 132.38 & 109.00 \\
1 & 26 & Vega & 557.36 & 4.83 & 8334.08 & 28.74 & 221.32 & 212.62 \\
3 & 27 & Vega & 426.53 & 4.04 & 8274.67 & 36.45 & 177.77 & 80.34
\end{tabular}

16 of 16 